\title{
From granulation hierarchy to granular perspective
}

\author{
C. Maria Keet \\ Faculty of Computer Science, Free University of Bozen-Bolzano, Italy. \\ keet@inf.unibz.it
}

\begin{abstract}
It is well-known that one can granulate data and information in multiple ways to generate a plethora of granulation hierarchies each with their levels of granularity. It is left implicit what the characteristics of such hierarchies are, and what consequences they have on levels of granularity. We propose a way to represent such additional information of granulation hierarchies by upgrading them to full granular perspectives and to provide a consistent way to uniquely identify, hence, distinguish, such perspectives based on their semantics by using a criterion and type of granularity used for granulation. In addition, with the chosen premises, definitions, and proven properties, we demonstrate some consequences for characterising levels of granularity within such granular perspectives.
\end{abstract}

\section{INTRODUCTION}

Granulating data, information, or knowledge results in the identification of granules, which are grouped into a level of granularity that in turn make up a granulation hierarchy. One can approach this with rough and fuzzy sets and logic as well as with the more traditional crisp semantics, where having a sound theory of the latter aids investigating details of the former two (see e.g., [12]). But how do we make different granulation hierarchies, what are its properties, how can we identify the hierarchies, and how can we manage those hierarchies in a consistent and reusable way in a granulated information system? We are interested here in hierarchies with levels such as cell $\prec$ tissue $\prec$ organ $\prec$ body, not the datainformation-knowledge abstraction levels [4], [17] (combining these two notions is a separate issue). Granularity lattices [3], [15] are basic structures that aim at representing granulation hierarchies, which can be a mere set of levels of detail [15] or indicated as "multiviews" [3] because each lattice presents a different perspective on the data. [11] introduces "granular world", a level, where its union is a "full granular space", which corresponds to a granulation hierarchy that always must be a taxonomy; it is unclear what the criteria are for unifying the granular worlds. [1] recognise hierarchies based on the parthood relation instead, but do not have a means to relate the hierarchies to each other. In praxis, however, people do want to link hierarchies, such as in GIS [2], [8], medicine [9], [13] and with ontologies for data integration in the Semantic Web for Life Sciences [14], where a requirement is to perform conditional selections with levels across hierarchies, such as 'for a map at the Country-level of granularity, show also the rivers with flow $\geq 100000 \mathrm{dm}^{3} /$ minute'. To achieve this, one needs to be able to identify hierarchies so as to enable distinguishing between them.

We aim to fill this gap by introducing the notion of granular perspective that provides a means of precisely representing the hitherto implicit characteristics of granulation hierarchies. To achieve this, we take a formal, ontology-inspired, approach, which enables us to identify and prove several properties of granular perspectives, such as that the perspective can be identified by the combination of criterion for granulation and the type of granularity chosen, that levels in the perspective have the same type of granularity, and that each level is in exactly one perspective. Not surprisingly, certain properties of the perspectives affect the notion of what granular levels are, which we shall address as well. While it may be possible to argue about a chosen ontological commitment, the purpose here is to demonstrate the consequences of such choices; we think that the characterisation of the essential properties of a granular perspective is quite elegant and, moreover, easy to implement regarding both the declarative part and for retrieving granulated information.

The remainder of the paper is structured as follows. After a brief overview in section II, we present the characterisation of a granular perspective in section III and consequences for granular levels in IV. We conclude in section V.

\section{BRIEF OVERVIEW}

The main components and their constraints that we will deal with in this paper are graphically depicted in Figure 1. The TypeOfGranularity that connects the types of granularity to perspective and level are summarised only up to the basic distinction between quantitative ( $\mathrm{SG}$ ) and qualitative (nG)

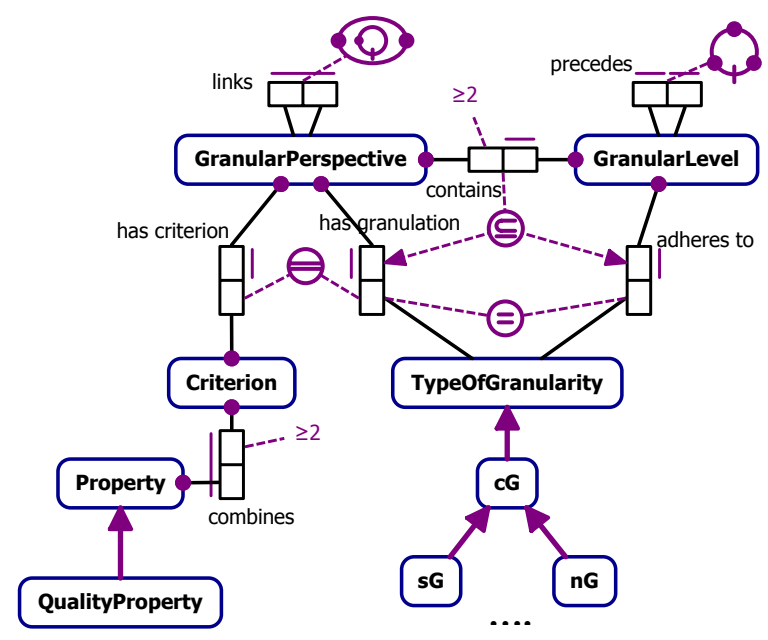

Fig. 1. Graphical depiction of the main entity types (roundtangles), their relations (rectangles), and constraints (purple icons) for granularity; see text for explanation and formalisation. 
granularity; see [5], [7] for a taxonomy with four quantitative and four qualitative leaf types of granularity. It is important to observe that these types of granularity each describe different mechanisms of granulation, such as using the parthood relation, multi-representation of an object, semantic aggregations (including taxonomies), and aggregating by fixed calculations (60 seconds in a minute, etc.). Then, for each granulation hierarchy to be consistent ontologically, exactly one of those types of granularity is used to devise the levels in the hierarchy; this is depicted with the blob and line next to the rectangle labelled with has granulation. Consequently, the levels in such a hierarchy adhere to the type of granularity used for constructing them. In addition, one needs a Criterion for selecting which properties of the objects are used to granulate and demarcate a section of the subject domain. These and other constraints will be proposed and proved in the next two sections. Due to space limitations, we shall not address all ontological considerations and justifications (such as why the precedes relation is at least a strict total order [6] with 1:1 participation constraint between the levels in a hierarchy [7]).

\section{IDENTIFYING GRANULAR PERSPECTIVES}

Although granular computing focuses primarily on granules and granular levels, granulating a body of data, information, or knowledge invariably results in a granulation hierarchy. Normally little knowledge about such hierarchies is described formally. However, there are various informal assumptions, such as the mechanisms of granulation, that ideally should be stated explicitly to make this implicit knowledge available for computational use. Henceforth, we shall call such a 'granulation hierarchy with additional properties' a granular perspective. For the notion of granular perspective, $G P$, one does not have to know which levels are in the perspective and how, but only that there are levels in the hierarchy; e.g., the perspectives human structural anatomy, modes of transmission of infectious agents [9], and administrative regions [2], [8]. None of these perspectives mention other aspects of the entities that are granulated, such as the functions of anatomical entities, the mode of action of the bacteria, and the size of the cities, respectively: these aspects are assumed to be dealt with in other perspectives. Put differently, when granulating, one highlights and chooses a view by using one or more properties along which to order the entities, but generally one does not use all properties of the entities to create a hierarchy with levels. Thus, when identifying or constructing a granulation hierarchy, one can use one or more particular attributes and group its values at different levels of detail or to use some other characteristic whilst ignoring other attributes; for instance, the grids of various sizes of cartographic maps and human structural anatomy (cells, tissues, organs, and so forth) that, in that hierarchy, ignore other properties of those entities such as a cell's function and the organ's spatial location. This basic notion of the usage of a selection of properties, noted elsewhere as well [1], [3], requires a closer ontological investigation into what kind of things those attributes and characteristics are. In philosophy, many kinds of properties have been identified [16]. For instance, a sortal property provides principles on identity (being a chair), an essential property is one where the individual always has that property for the time of the individual's existence (being cat is an essential property of Garfield), natural (protein) and artificial (television) kinds, and extrinsic and intrinsic properties. Which of those kinds of properties are, or should be used, for granulation, or if any of them is fine, requires more ontological investigation. For the time being, we simplify this to the fact that for granularity it is important that one does granulate according to specific properties with which the domain is partitioned, levels identified, and subject domain granulated. Looking ahead to computational use, it demands for a way to formally represent it. Knowledge representation and software engineering are flexible about how to formally represent properties, such as attributes in a UML Class diagram or as unary or binary predicates. For our purpose, we can generalise from this and use the criterion for granulation. This criterion for granulation, $C$, is a combination of either at least two properties, Prop, or at least one property and a quality property, $Q$ where $\forall x(Q(x) \rightarrow \operatorname{Prop}(x))$, that has a measurable region. The idea behind the distinction between Prop and $Q$ is to have a means to represent the difference between qualitative and quantitative granularity. For any level that adheres to the quantitative $\mathbf{S G}$ type of granularity, or one of its subtypes, the value or value range is determined by the type of scale used; e.g., Surface (a Prop) and Surface metric (a $Q$ ) with three levels $l_{1}, l_{2}$, and $l_{3}$ can have the values $\mathrm{km}^{2}, \mathrm{hm}^{2}$, and dam ${ }^{2}$, respectively (recollect that $l_{3} \prec l_{2} \prec l_{1}$ is valid, which does not imply that there is a subclass relation between either the levels or its contents: $\mathrm{dam}^{2}$ is not a taxonomic subtype of $\mathrm{km}^{2}$ but a proper part of $\mathrm{km}^{2}$ ). Thus, the semantics of such as scale is part of a granulation criterion and can be housed in the QualityProperty. We use has_value $(x, y)$ (Definition 1 and Proposition 1$)^{1}$ for a means to record the values and we note the value's upward distributivity from property to its criterion (Proposition 2).

Definition 1 (has_value): The has_value relation relates a property with its value: $\forall x, y\left(h a s \_v a l u e(x, y) \rightarrow \operatorname{Prop}(x) \wedge\right.$ $V(y))$.

Proposition 1: Each quality property $Q(x)$ has some value $V(y)$, which is related through the relation has_value $(x, y)$ : $\forall x\left(Q(x) \rightarrow \exists y\left(h a s \_v a l u e(x, y)\right)\right)$.

Proposition 2: By upward distributivity, value(s) of the property/ies Prop and/or $Q$ of the criterion are also values of the criterion $C: \forall x, y($ has_value $(x, y) \rightarrow$ $\left.\exists z\left(h a s \_v a l u e(z, y) \wedge C(z)\right)\right)$.

For qualitative granularity-i.e., nG and its subtypesthe amount of properties considered at a finer-grained level increases (e.g., with respect to taxonomic subsumption); that is, any criterion $C$ will not provide a single obvious property with changing numerical values for non-scale-dependent levels

\footnotetext{
${ }^{1}$ has_value $(x, y)$ corresponds in spirit to " $q l$ " in DOLCE [10] foundational ontology.
} 
across the hierarchy. For instance, in the straightforward perspective of human structural anatomy, we have, e.g., $l_{i}=$ Organ and $l_{j}=$ Cell without an obvious distinctive value other than a change in name and not using a measurement. Either way, we need a way to relate those properties that combine into a criterion it is used for, $C P$ (Definition 2), and use that relation in a basic definition of criterion $C$ in Definition 3.

Definition $2(C P)$ : The relation $C P$ relates a criterion $C$ to the properties it combines: $\forall x, y(C P(x, y) \rightarrow C(x) \wedge$ Prop $(y))$, where there are at least two properties participating: $\forall x\left(C(x) \rightarrow \exists^{\geq 2} y C P(x, y)\right)$

Definition 3 (Criterion): Each criterion $C$ is a combination of either at least two properties Prop but not a quality property $Q$, i.e., $\exists^{\geq 2} y(\operatorname{Prop}(y) \wedge \neg Q(y))$, or at least one Prop and exactly one $Q$, i.e., $\exists y \exists ! z(\operatorname{Prop}(y) \wedge Q(z) \wedge \neg(y=z))$. which are related to $C$ through the $C P$ relation.

Following from Definition 3 and the types of granularity, when a $Q$ is used for a $C$ then we deal with scale-dependent granularity (Proposition 3).

Proposition 3: If a criterion $C$ has at least one Prop and exactly one $Q$, then it is associated with granulation type $\boldsymbol{s} \boldsymbol{G}$.

The criterion $C$ provides the what is to be granulated in addition to the how provided by the TypeOfGranularity $(T G)$. These two components have to be related to GranularPerspective, $G P$, before defining granular perspective. The former is done through $R C$ (read: has criterion, Definition 4) and the latter through $R G_{p}$ (read: has granulation, Definition 5) where the greek letters are syntactic sugar for the eight leaf types of granularity (i.e., a finite list of first order axioms so we remain within FOL).

Definition $4(R C)$ : Relation $R C(x, y)$ holds between perspective $G P(x)$ that has criterion $C(y): \forall x, y(R C(x, y) \rightarrow$ $G P(x) \wedge C(y))$.

Definition $5\left(R G_{p}\right)$ : The relation $R G_{p}(x, \phi)$ holds if $G P(x)$ and $T G(\phi)$ where $T G$ is the type of granularity: $\forall x, \phi\left(R G_{p}(x, \phi) \rightarrow G P(x) \wedge T G(\phi)\right)$.

In addition to the basic typing of the relations, several constraints can be added. First, we add a mandatory (total) participation to $R C$, because there is no reason to have a criterion for granulation in an information system without actually using it (Proposition 4). Second, one can neither use more than one criterion for one perspective nor use none (because then there is nothing to granulate), therefore we add proposition Proposition 5. The intuition of this proposition is that, ontologically, it is nonsense to combine, say, criterion $c_{1}=$ Human pathological processes at different levels of granularity with $c_{2}=$ Mouse structural anatomy at different levels of granularity to make one single hierarchy of levels.

Proposition 4: Each criterion must participate in a $R C$ : $\forall x(C(x) \rightarrow \exists y R C(y, x))$.
Proposition 5: Each perspective has exactly one criterion: $\forall x(G P(x) \rightarrow \exists ! y R C(x, y))$.

Recollecting one always uses a type of granularity for granulating the data, we thus have a mandatory participation of $G P$ in the $R G_{p}$ relation, because if one does not use a type of granularity at all, then one does not granulate as it would negate any granular structure among entities. In addition, one should not mix different ways of granulating data within one perspective lest the hierarchy of levels will be inconsistent; hence combining two or more types leads to a contradiction. Thus, each perspective has exactly one $T G$ :

Lemma 1: Each perspective has exactly one type of granulation: $\forall x\left(G P(x) \rightarrow \exists ! \phi R G_{p}(x, \phi)\right)$.

With this characterisation, denoting with $D^{f}$ the entity that contains all the explicitly defined granular perspectives to granulate the subject domain, and using the notions of concept $(C N)$ and definition $(D F)$ from the DOLCE foundational ontology [10], we arrive at a preliminary definition-list of properties-of $G P$.

Definition 6 (Granular perspective [6]): $\forall x \exists ! w, y, z, \phi$ such that $G P(x)$ is a concept $C N(x)$, has a definition $D F(x, y)$, relates to its criterion $C(z)$ through the relation $R C(x, z)$, has granulation, $R G_{p}$, of type $T G(\phi)$ and is contained in a domain $D^{f}(w)$.

Following from the definitions and propositions, Lemma 2identifying a path between $C$ and $T G$ through $G P$ - can be proved now.

Lemma 2: If $C(x)$ has a $Q(y)$ and $R C(z, x)$, then that $G P(z)$ has granulation type $\mathbf{s}: \forall x \exists z, \phi((C(x) \rightarrow$ $\left.\exists ! y(C P(x, y) \wedge Q(y))) \wedge R C(z, x) \wedge R G_{p}(z, \phi) \rightarrow(\phi \rightarrow s G)\right)$.

Proof: First, Definition 3 can be formalised as $\forall x\left(\left(C(x) \quad \rightarrow \quad \exists^{\geq 2} y(\operatorname{Prop}(y) \wedge \neg Q(y))\right) \underline{\vee}(C(x) \rightarrow\right.$ $\exists y \exists ! z(\operatorname{Prop}(y) \wedge Q(z) \wedge \neg(y=z)))$

Given we have a $Q$, then the second part after the exclusive-or in Definition 3 must hold. Second, we have the typing of $R C$ and mandatory constraint

$\forall x, y(R C(x, y) \rightarrow G P(x) \wedge C(y))$

(Definition 4)

$\forall x(C(x) \rightarrow \exists y R C(y, x))$

(Proposition 4)

therefore, there has to be an instance, $a$, of $G P$ (first argument in $R C$ ). Given this instance $a$ and

$\forall x, \phi\left(R G_{p}(x, \phi) \rightarrow G P(x) \wedge T G(\phi)\right)$

$\forall x\left(G P(x) \rightarrow \exists ! \phi R G_{p}(x, \phi)\right)$

(Definition 5)

(Lemma 1)

therefore, there must be a $\phi$ that is a $T G$. By having $Q$ (first point) and Proposition 3, then $\phi=s G$, therefore $G P(z)$ has granulation type $\mathbf{s G}$.

From the proof of Lemma 2 it follows immediately that the other half of the definition of $C$ applies to $\mathbf{n G}$ (Corollary 1), due to the exclusive-or in Definition 3 and disjoint subtypes in the taxonomy of types of granularity.

Corollary 1: If $C(x)$ has $\geq 2$ properties $\operatorname{Prop}(y)$ and $\neg Q(y)$, then $G P(z)$ has granulation type $\boldsymbol{n} \boldsymbol{G}$. 
Now we add an interesting property of granular perspectives concerning reuse of criteria (Lemma 3), from which follows that the combination of criterion and type of granulation determines uniqueness of a GP (Theorem 1); hence, together they provide the necessary and sufficient conditions for identity of GP. The Prover9computed proofs for Lemma 3 and Theorem 1 are online at [http://www.meteck.org/files/grc09computedproofs.zip].

Lemma 3: A criterion $C$ can be used with more than one perspective $G P$, provided the perspectives have distinct granulation types $T G: \forall x_{1}, x_{2}, y, \phi_{1}, \phi_{2}\left(R C\left(x_{1}, y\right) \wedge R C\left(x_{2}, y\right) \wedge\right.$ $\left.R G_{p}\left(x_{1}, \phi_{1}\right) \wedge R G_{p}\left(x_{2}, \phi_{2}\right) \wedge \neg\left(x_{1}=x_{2}\right) \rightarrow \neg\left(\phi_{1}=\phi_{2}\right)\right)$.

Proof: For each $G P$ we have a $C(y)$ and a $T G(\phi)$, because of

$\forall x(G P(x) \rightarrow \exists ! y R C(x, y))$

(Proposition 5)

$\forall x\left(G P(x) \rightarrow \exists ! \phi R G_{p}(x, \phi)\right)$

(Lemma 1)

Assume for some $y$, i.e., instance $c_{1} \in C$, and some $\phi$, there is the same instance of $x, p_{1} \in G P$, i.e., $R C\left(p_{1}, c_{1}\right)$ and $R G_{p}\left(p_{1}, \phi\right)$ hold too. Let us reuse $\phi$ for some other perspective, $p_{2} \in G P$, so that $R G_{p}\left(p_{2}, \phi\right)$ and assume $p_{2} \neq p_{1}$ hold. Let us also reuse $c_{1}$ for some other perspective, $p_{3} \in$ $G P$, i.e., $R C\left(p_{3}, c_{1}\right)$ and assume $p_{3} \neq p_{1}$ hold. Then we have two cases:

(i) $p_{3}=p_{2}$ : then by Proposition 5 and Lemma 1 either $p_{3}=p_{2}=p_{1}$ (thus contradicting the assumptions $p_{2} \neq p_{1}$ and $p_{3} \neq p_{1}$ ) or there is an elusive property $\alpha$ to negate the equality. There is no $\alpha$, hence, it must lead to identity of $G P$ with $C$ and $T G$. Thus,

$\forall x_{1}, \ldots, x_{4}, y_{1}, y_{2}, \phi_{3}, \phi_{4}\left(R C\left(x_{1}, y_{1}\right) \wedge R C\left(x_{2}, y_{2}\right) \wedge\right.$ $R G_{p}\left(x_{3}, \phi_{3}\right) \wedge R G_{p}\left(x_{4}, \phi_{4}\right) \wedge y_{1}=y_{2} \wedge \phi_{3}=\phi_{4} \rightarrow$ $\left.x_{1}=x_{2}=x_{3}=x_{4}\right)$.

(ii) $p_{3} \neq p_{2}$ : then by Lemma 1 , we have $R G_{p}\left(p_{3}, \phi^{\prime}\right)$ and $\phi \neq \phi^{\prime}$, and by Proposition 5, we have $R C\left(p_{2}, c_{2}\right)$ and $c_{1} \neq c_{2}$.

Thus, reuse of criterion $c_{1}$ with another $T G, \phi^{\prime}$, is demonstrated in point (ii) with $p_{3}$.

Theorem 1: The combination of some $C(y)$ with a $T G(\phi)$ determines uniqueness of each $G P(x)$.

Proof: Follows from Lemma 3, point (i).

For instance, we can have a $c_{i}=$ Mouse structural anatomy that can be granulated according to different mechanisms, such as by a partonomy $(\phi)$ and as a taxonomy $\left(\phi^{\prime}\right)$, so that we have two different granular perspectives. From Lemma 3 and Theorem 1 it trivially follows that for $D^{f}$, the perspectives are unique (Corollary 2), where $R E$ denotes the relation between $D^{f}$ and the perspectives (see below).

Corollary 2: Granular perspectives are unique within the domain they are contained in: $\forall x_{1}, \ldots, x_{n}, y\left(G P\left(x_{i}\right) \wedge D^{f}(y) \wedge\right.$ $\left.R E\left(x_{i}, y\right) \rightarrow \neg\left(x_{1}=x_{2}\right) \wedge \ldots \wedge \neg\left(x_{n-1}=x_{n}\right)\right)$.

Put differently, all perspectives $p_{1} \ldots p_{n} \in G P$ contained in a $D^{f}$ are disjoint. Observe that one cannot derive a complete coverage unless one were to take a closed-world assumption and assume that all entities in the represented subject domain must be granulated.

It is ontologically more appropriate and representationally more convenient to use the notion of $D^{f}$ compared to a simple set of perspectives (see e.g., [7]) and to explicitly relate that to the perspectives with the relation $R E$. Also, it is practically useful in the light of information system integration. In addition, looking ahead to relating level to perspective in the next section, we will be able to use the same relation $R E$. A primitive definition is as follows, where proper parthood is defined in terms of parthood in the usual way.

Definition $7(R E)$ : For all $x$ there exists a $y$ where the relation $R E(x, y)$, and its inverse $R E^{-}$, holds between two of the three granularity components iff

- $G L(x) \wedge G P(y)$ or $G P(x) \wedge D^{f}(y)$ for $R E(x, y)$, and

- $D^{f}(x) \wedge G P(y)$ or $G P(x) \wedge G L(y)$ for $R E^{-}(x, y)$.

Further, $R E(x, y) \rightarrow$ ppart_of $(x, y)$ and $R E^{-}(x, y) \rightarrow$ has_ppart $(x, y)$.

Last, we can relate the granular perspectives to each other in various ways so as to, ultimately, link granular levels of different perspectives and retrieve additional multi-granular information. This notion has not been addressed in [1], [3], whereas [7] proposes an elaborate mereology-based approach as well as a simple one that corresponds to the links relation in Figure 1. Such a 'simple' relation, denoted here with $R P$, can be typed as shown in Definition 8 , from which it follows immediately that $R P$ is irreflexive and symmetric (Lemma 4).

Definition $8(R P): R P$ relates two distinct perspectives: $\forall x, y(R P(x, y) \rightarrow G P(x) \wedge G P(y) \wedge \neg(x=y))$.

Lemma 4: $R P$ is irreflexive, $\neg R P(x, x)$, and symmetric, $R P(x, y) \leftrightarrow R P(y, x)$.

Proof: Irreflexive: the " $\neg(x=y)$ " in Definition 8 and one or more unique perspectives (Corollary 2), therefore the relata can never be the same. Symmetric: $R P$ 's distinct domain and range are both of type $G P$.

One might want to refine this definition to also include a 'swapping' of criteria, but from previous results on properties of granular levels, it was shown that it is the combination of criterion and granulation what makes a perspective unique (Theorem 1), hence, shifting perspective already logically implies changing $C$ or $T G$. Thus, a relation between perspectives within a domain suffices for the current scope, where the resultant of switching is that different properties of the granulated contents will be highlighted. $R P$ is necessary when we need to link levels from different granular perspectives, whereby we can retrieve additional targeted information through using $R P$ not possible with the elaborate mereology-based approach. For instance, let $d_{i}^{f}=$ Infectious Diseases [9], Vibrio cholerae located at the Species-level $l_{7}$ in perspective $p_{1}=$ Taxonomy and in $l_{3}=$ Inhibitor of a $p_{2}=$ Pathological mode of action. Using the relations between the levels in the hierarchies as well 
as $R P$, one can pick up information along the path, thereby retrieving more knowledge by taking advantage of granularity to a greater extent; in casu, that at the coarser-grained $l_{1}$ of $p_{1}, V$. cholerae is a Bacterium and of the pathology $p_{2}$ in level $l_{1}$ a Toxin-producer.

This concludes the initial characterisation and means for identification of granular perspectives. Such represented knowledge has an effect on the notion of granular levels and what information about levels one can represent. This is the topic of the next section.

\section{TOWARD CHARACTERISING GRANULAR LEVELS}

The characterisation of granular perspective compared to a mere granulation hierarchy has an effect on what it contains. Analogous to the former, we can say that a granular level ( $G L$, for short) is 'something more' than merely a collection of granules. The specification of a level in a particular subject domain is relevant only after knowing the criterion and type of granulation. $G L$ delimits what it is to be a level and of a certain level and, analogous to $G P$, has a definition and constraints, and is a concept, too. If one has a granular level, there must be a perspective it is contained in, lest one creates levels freely by combining types of granularity or mixing criteria that would result in inconsistent granulation. For this purpose, we can reuse the $R E$ relation introduced earlier (Definition 7) and add a mandatory participation in $R E$ by $G L$ (Proposition 6 ).

Proposition 6: For all $x$, where $G L(x), x$ is contained in a granular perspective: $\forall x(G L(x) \rightarrow \exists y(R E(x, y) \wedge G P(y)))$.

In fact, based on indistinguishability and similarity [6], Proposition 6 can be constrained further to have at least two granular levels in a granular perspective $(\forall x(G P(x) \rightarrow$ $\left.\exists \geq 2 y\left(R E^{-}(x, y) \wedge G L(y)\right)\right)$ so as to have a a means to record things at different levels of detail. In addition, one does not have to redefine the criterion for granulation for each granular level, because this is already taken care of by its $G P$ 's criterion $C$, but the values of $G P$ 's criterion are needed to distinguish between different levels in a perspective and to establish that no two levels are identical in one granular perspective.

With a tentative, minimal characterisation of granular level, we already can prove some additional properties, such as that each level can occur only once in a perspective and that it must adhere to the same type of granularity as its perspective. These properties of a granular level conceptually follow from both the notion of granular perspective and notions such as indistinguishability and similarity [6]. It does not preclude one from identifying and adding more properties or attributes to the notion of granular level. Here, we first add a relation for $G L$ that, like the granular perspective, it also relates to a type of granularity, $T G$, which we realise with the adheres to relation, abbreviated in the formalisms with $R G_{l}$ (Definition 9), which has an additional mandatory constraint to ensure the type of granularity constrains the structure of the contents of that level (Proposition 7).
Definition $9\left(R G_{l}\right)$ : The relation $R G_{l}(x, \phi)$ holds if $G L(x)$ and $T G(\phi)$, i.e., $\forall x, \phi\left(R G_{l}(x, \phi) \rightarrow G L(x) \wedge T G(\phi)\right)$.

Proposition 7: Each GL must adhere to a TG: $\forall x\left(G L(x) \rightarrow \exists \phi R G_{l}(x, \phi)\right)$.

Now we have sufficient ingredients to provide a basic, preliminary version of a definition for granular level. Like with the definition for granular perspective, several categories from DOLCE [10] are used, being concept $C N$, definition $D F$, quality $Q$, and region $V$. In addition, has_value $(x, y)$ (Definition 1) and $R E(x, y)$ (Definition 7) are reused.

Proposition 8 (Granular level (preliminary version)): $\forall x \exists ! v, w, y, z \exists p$ such that $G L(x)$ is a concept $C N(x)$, has a definition $D F(x, y)$, is related to $G P(w)$ with $R E(x, w)$ and uses criterion $C(z)$ with $R C(w, z)$ and has_value $(z, v)$ where the value is in region $V(v)$ for any $G L(x)$ that adheres_to $\boldsymbol{s} \boldsymbol{G}, G L^{s}(x)$, and $z$ 's label for any $G L(x)$ that adheres_to type $\boldsymbol{n G}, G L^{n}(x)$. Entities residing in $G L^{s}(x)$ are similar to each other with respect to (the value $z$ of) $V(v)$, entities residing in $G L^{n}(x)$ are similar to each other with respect to (the label of the universal of) $\operatorname{Prop}(p)$ of $C(z)$, and both are $\varphi$-indistinguishable with respect to its adjacent coarser-grained level.

Given this basic characterisation and the above-defined and proven characteristics, we can prove several additional properties. The "role subset" (encircled " $\subseteq$ ") and "role equality" (encircled "=") constraints shown in Figure 1 will be proven first; that is, Lemma 5 does not ensure GP and its $G L$ use the same $T G$ because the " $\exists \phi$ " says there is at least one of them, but to achieve this, we need Lemma 6 .

Lemma 5: For each $G P(x)$ and $G L(y)$ over their join paths, the following holds: if $G P(x)$ contains $G L(y)$, then $G P(x)$ has granulation some $T G$ and $G L(y)$ adheres to some $T G$ :

$$
\begin{gathered}
\forall x, y(R E(x, y) \wedge G P(y) \wedge G L(x) \rightarrow \\
\left.\exists \phi\left(R G_{p}(y, \phi) \wedge R G_{l}(x, \phi)\right)\right)
\end{gathered}
$$

Proof: First, given

$\forall x(G L(x) \rightarrow \exists y(R E(x, y) \wedge G P(y)))$

(Proposition 6)

$\forall x\left(G P(x) \rightarrow \exists^{\geq 2} y\left(R E^{-}(x, y) \wedge G L(y)\right)\right) \quad$ (Thm 1 in [6]) therefore, if we have a $G P$, then there must be $\geq 2$ instances of $G L$ related to it and if we have a $G L$ that there must be a $G P$. Assume $a, b$ such that $G P(a)$ and $G L(b)$, then with $\forall y\left(G P(y) \rightarrow \exists ! \phi R G_{p}(y, \phi)\right)$ $\forall x\left(G L(x) \rightarrow \exists \phi R G_{l}\left(x, \phi^{\prime}\right)\right)$

(Lemma 1) either $\phi=\phi^{\prime}$ or $\phi \neq \phi^{\prime}$ so that there must be $\geq 1$ TG and therefore (1) holds.

Lemma 6: For each TG, some $G L(x)$ adheres to that $T G$ if and only if some $G P(y)$ $R G_{p}$ that $T G: \forall \phi\left(\exists y R G_{p}(y, \phi) \leftrightarrow \exists z R G_{l}(z, \phi)\right)$.

Proof: Assume GP and GL are (mutually dependent) instantiated so that they must have a TG (Lemma 5). Given Lemma 1 and that each structure of level contents of the leaf types are distinct, then also $\forall x\left(G L(x) \rightarrow \exists ! \phi R G_{l}\left(x, \phi^{\prime}\right)\right)$ 
must hold, because combining two or more types leads to a contradiction. Further, from Proposition 8 we have "uses criterion $C(z) \ldots$.." and by

$\forall x(G P(x) \rightarrow \exists ! y R C(x, y))$

(Proposition 5)

$R E$ relating $G L$ to its $G P$, having

$\forall x\left(G P(x) \rightarrow \exists ! y, \phi\left(R C(x, y) \wedge R G_{p}(x, \phi)\right)\right) \quad$ (Theorem 1$)$

and aforementioned Lemma 1, therefore, the $G L$ uses the same criterion as its $G P$, hence $\phi=\phi^{\prime}$ holds, too.

The combination of Lemma 5 and Lemma 6 can be formulated in a shorter constraint:

$$
\begin{gathered}
\forall x, y(G P(y) \wedge G L(x) \wedge R E(x, y) \rightarrow \\
\left.\exists ! \phi\left(R G_{p}(y, \phi) \leftrightarrow R G_{l}(x, \phi)\right)\right)
\end{gathered}
$$

With these results obtained, we can strengthen Proposition 6 and prove that each $G L$ is contained in exactly one $G P$ (Theorem 2) (the Prover9-computed proof is online at [http://www.meteck.org/files/grc09computedproofs.zip]):

Theorem 2: For all $x$, where $G L(x), x$ is contained in exactly one granular perspective: $\forall x(G L(x) \rightarrow \exists ! y R E(x, y))$.

Proof: We already have at-least-one $G L$ in $G P$ (Proposition 6) and need to demonstrate the at-most-one $(R E(x, y) \wedge R E(x, z) \rightarrow y=z)$. GL uses the $C$ of $G P$ it is contained in (Proposition 8), which still permits a $G L$ to be reused in another $G P$. However, $G L$ adheres to the same $T G$ as its $G P$ it is contained in (axiom (2)). Given

$\forall x_{1}, \ldots, x_{4}, y_{1}, y_{2}, \phi_{3}, \phi_{4}\left(R C\left(x_{1}, y_{1}\right) \wedge R C\left(x_{2}, y_{2}\right) \wedge\right.$ $R G_{p}\left(x_{3}, \phi_{3}\right) \wedge R G_{p}\left(x_{4}, \phi_{4}\right) \wedge y_{1}=y_{2} \wedge \phi_{3}=\phi_{4} \rightarrow x_{1}=$ $\left.x_{2}=x_{3}=x_{4}\right)$

(Theorem 1)

$\forall x_{1}, \ldots, x_{n}, y\left(G P(x) \wedge D^{f}(y) \wedge R E(x, y) \rightarrow \neg\left(x_{1}=\right.\right.$ $\left.\left.x_{2}\right) \wedge \ldots \wedge \neg\left(x_{n-1}=x_{n}\right)\right) \quad$ (Corollary 2) there cannot be another $G P$ with the same $C$ and $T G$ in one $D^{f}$, hence, $G L$ can be $\leq 1$ time in a perspective. Thus, $\geq 1$ and $\leq 1$ is exactly one, i.e., $\forall x(G L(x) \rightarrow \exists$ !yRE $(x, y))$

With the characteristics of levels and perspectives, one can proceed further to assess if the type of granularity permits or requires additional properties of granular levels. This is indeed the case for quantitative granularity. For instance, the values of a level's usage of criterion is more encompassing that that of its adjacent finer-grained level for those levels that adhere to $\mathbf{S G}$ type of granularity and we can relate a function to such granular levels to be used for 'converting' contents of one level into its adjacent coarser level or vice versa-e.g., $60 * 1$ minute $=1$ hour - and that there are $\leq 2$ mathematical functions associated to a granular level to take care of the conversions between these values; due to space limitations, the human-readable proofs of these simple additions can be found in [7] and Prover9-computed proof at [http://www.meteck.org/files/grc09computedproofs.zip]. From an engineering point of view, a maximum of two functions for each level may seem prohibiting, but that is, theoretically, all one requires for traversing $\mathbf{s G}$-granulated levels. Any other granularity conversion function to, say, skip a level for aggregating data, are extras to, e.g., improve database performance, but this is outside the theoretical need. We are currently investigating the precise needs for such additional functions that can enhance usability and performance.

\section{CONClusions}

We have demonstrated a mechanism for representing granular perspectives, including identifying them through the unique combination of criterion and its type of granularity used for granulation. In addition, we have demonstrated some consequences for characterising levels of granularity within such granular perspectives, such as that those levels must adhere to the same type of granularity as their perspective and that each level is in exactly one perspective. Given that our aim is to enhance granulated information systems, we are currently investigating how this can be captured best in a computationally well-behaved fragment of first order logic.

\section{REFERENCES}

[1] T. Bittner and B. Smith, Foundations of Geographic Information Science. London: Taylor \& Francis Books, 2003, ch. A Theory of Granular Partitions, pp. 117-151.

[2] E. Camossi, M. Bertolotto, E. Bertino, and G. Guerrini, "Issues on modelling spatial granularity," in Workshop on fundamental issues in spatial and geographic ontologies, 2003, 23 Sept. 2003. Ittingen, Switzerland.

[3] Y. H. Chen and Y. Y. Yao, "Multiview intelligent data analysis based on granular computing," in IEEE International Conference on Granular Computing (GrC2006). IEEE Computer Society, 2006, pp. 281-286.

[4] B. Edmonds, "Complexity and scientific modelling," Foundations of Science, vol. 5, no. 3, pp. 379-390, 2000.

[5] C. M. Keet, "A taxonomy of types of granularity," in IEEE International Conference on Granular Computing (GrC2006). IEEE Computer Society, 2006, pp. 106-111.

[6] _- "Granulation with indistinguishability, equivalence or similarity," in IEEE International Conference on Granular Computing (GrC2007), vol. 2. IEEE Computer Society, 2007, pp. 11-16.

[7] _ "A formal theory of granularity," $\mathrm{PhD}$ Thesis, KRDB Research Centre, Faculty of Computer Science, Free University of Bozen-Bolzano, Italy, April 2008.

[8] — "Structuring GIS information with types of granularity: a case study," in Proceedings of the 6th International Conference on Geomatics, 2009, la Habana, Cuba, Feb 10-12, 2009.

[9] C. M. Keet and A. Kumar, "Applying partitions to infectious diseases," in Connecting Medical Informatics and bio-informatics (MIE'05). Amsterdam: IOS Press, 2005, pp. 1236-1241.

[10] C. Masolo, S. Borgo, A. Gangemi, N. Guarino, and A. Oltramari, “Ontology library,” WonderWeb Deliverable D18 (ver. 1.0, 31-12-2003), 2003, http://wonderweb.semanticweb.org.

[11] T. Qiu, X. Chen, Q. Liu, and H. Huang, "A granular space model for ontology learning," in IEEE International Conference on Granular Computing ( $G r C^{\prime}$ '07). IEEE Computer Society, 2007, pp. 61-65.

[12] M. Reformat and C. Ly, "Ontological approach to development of computing with words based systems," International Journal of Approximate Reasoning, vol. 50, pp. 72-91, 2009.

[13] B. Ribba, T. Colin, and S. Schnell, "A multiscale mathematical model of cancer, and its use in analyzing irradiation therapies," Theoretical Biology and Medical Modeling, vol. 3, p. 7, 2006.

[14] B. Smith et al., "The OBO Foundry: Coordinated evolution of ontologies to support biomedical data integration," Nature Biotechnology, vol. 25, no. 11, pp. 1251-1255, 2007.

[15] J. Stell and M. Worboys, "Stratified map spaces: a formal basis for multi-resolution spatial databases," in Proceedings of the 8th International Symposium on Spatial Data Handling (SDH'98). International Geographical Union, 1998, pp. 180-189.

[16] C. Swoyer, "Properties," in The Stanford Encyclopedia of Philosophy, winter 2000 ed., E. N. Zalta, Ed. Stanford, 2000, http://plato.stanford.edu/archives/win2000/entries/properties/.

[17] Y. Yao, Human-Centric Information Processing Through Granular Modelling. Springer, 2009, ch. Integrative Levels of Granularity, pp. 31-48. 